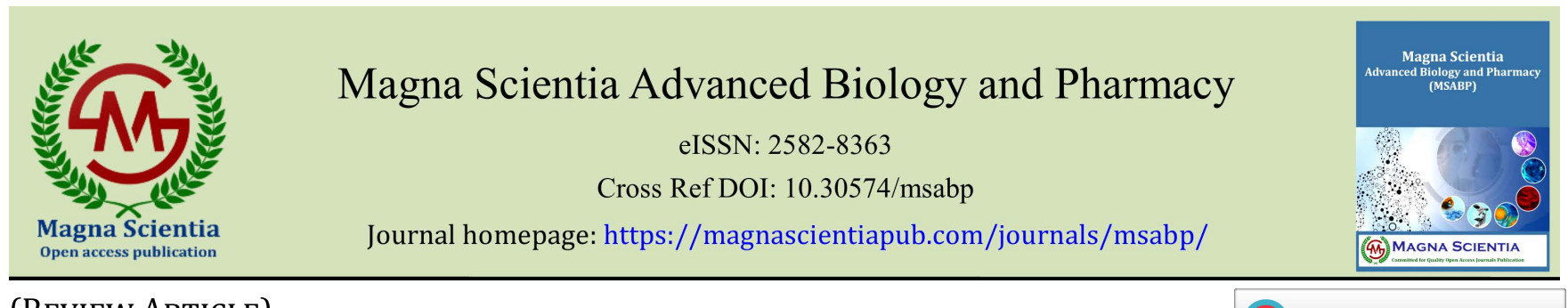

(REVIEW ARTICLE)

\title{
Foot and mouth disease virus: A review
}

\author{
Rawaa S. Jumaa *, Sabrin I. Mohsin, Dhuha I. Abdulmjeed and Osama F Atshan \\ Department of Microbiology of Veterinary Medicine, College of Veterinary Medicine, University of Baghdad, Iraq.
}

Magna Scientia Advanced Biology and Pharmacy, 2021, 03(02), 027-035

Publication history: Received on 29 July 2021; revised on 06 September 2021; accepted on 08 September 2021

Article DOI: https://doi.org/10.30574/msabp.2021.3.2.0038

\begin{abstract}
As seen by prior tragic outbreaks in many places throughout the world, the foot and mouth disease virus, or "FMDV," is one of the most critical challenges in animal health. In this review, the major features of FMDV, as well as aspects of its interactions with cells and hosts, were discussed. On the other hand, present and upcoming FMD treatment approaches. The first vertebrate virus found was the foot-and-mouth disease virus (FMDV). A capsid protein and the viral genome (+ve sense single strand RNA) make up FMDV. The icosahedral symmetry of the viral structure is made up of structural proteins (VP1, VP2, VP3, and VP4) as well as non-structural proteins (L, 1A, 1B, 1C, 1D, 2A, 2B, 2C, 3A, 3B, 3C, and 3D). The viral replication takes place in the cytoplasm of the cell. Because FMDV has a short incubation period, it spreads quickly. Direct contact is the most often used method of FMDV transmission. The occurrence of direct contact via aerosol and mechanical transmission (fomites, feed, and water). The immunological response is stimulated by the infection with FMD. However, due to virus antigenic diversity, the immune response does not always protect against FMD (antigenic shift). FMDV is divided into seven serotypes based on antigenic variation. 0, A, C, SAT-1, SAT-2, SAT-3, and Asia-1 are the serotypes in question. 0 is the most frequent serotype.
\end{abstract}

Keywords: Foot and mouth disease; Structure; Replication; Epidemiology; Pathogenesis; Diagnosis and Vaccines

\section{Introduction}

Foot and mouth disease virus was the first animal virus found. It consists of 8500 base-positive-sense, a single-stranded RNA genome, arranged into three main parts: a small, poly-adenylated $3^{\prime}$ end and linked protein at the $5^{\prime}$ end. Because of the short in vivo replication cycle and sudden start of shedding, FMDV outbreaks can spread quickly between and within populations [1]. Direct contact is the most prevalent method of FMDV infection; nevertheless, aerosol and mechanical viral dispersion through water, feed, and fomites can result in infection over great distances. Unvaccinated and vaccinated ruminants both have FMDV carriers who are medically sound, hindering control and elimination efforts. The infection triggers a rapid immune response, although protection to one FMDV isolation may not consistently protect against others according to antigenic variation. Antigenic variation complicates FMDV control and has the prospective to complicate vaccinology and diagnostics $[2,3]$.

Clinical diseases have been managed with inactivated serotype-specific FMDV vaccinations but in "FMDV" eradication campaigns, as well. in endemic areas. Researchers have tried to develop FMDV vaccines on calves, pig kidneys, porcine kidney or baby hamster kidney fibroblast by utilizing formaldehyde Inactivated Fluid and loose epithet tissue on veins, baby hamster kidneys. One of these large-scale cell lines employed in the production of FMDV vaccine is BHK-21[4]. "ADIVA" technique based on antibodies against NSPs in infected animals is used to control the effective implementation of FMDV eradication and to preserve the status of "FMD" free of immunization. "FMDV" vaccinations have, nevertheless, been discovered to include sufficient none structural protein "NSP" to activate "anti-NSP" that lead to fake positive results of the immunosorbent test results associated with enzymes. Whether the aim is early detection, long-term

\footnotetext{
${ }^{*}$ Corresponding author: Rawaa Saladdin Jumaa E-mail: firasrashad@gmail.com

Department of Microbiology of Veterinary Medicine, College of Veterinary Medicine, University of Baghdad, Iraq. 
monitoring or elimination, diagnostic, analytical sensitivity and particular techniques to monitor FMDV are necessary $[5,6]$. In this paper, the categorization, structure, genome and diagnostic specimens of FMDV are reviewed.

\subsection{Causes of FMD}

The FMDV is the Picornaviridae family member and the Aphthovirus prototypical genus member. "Equine rhinitis A virus", once known as "equine rhinovirus 1", has a more FMDV-like genomic structure than other picornaviruses. FMDV is very susceptible to genetic and antigenic variation, which has led to the classification among circulating viruses of seven serotype FMDV "A, 0, C, Asia1, SAT 1, SAT 2, and SAT 3)" based on their capability of inducing cross-protection in animals [7].

\subsection{Viral Structure of Aphthovirus}

$\mathrm{T}=\mathrm{icosahedral,} \mathrm{"non-enveloped",} \mathrm{spherical,} \mathrm{around} \mathrm{"30} \mathrm{nm"} \mathrm{in} \mathrm{the} \mathrm{diameter,} \mathrm{pseudo} 3$ around the "naked RNA genome". The capsid consists of 60-protomer icosahedral organisation. The interior of the capsid is composed of four polypeptides, "VP1, VP2, VP3 and VP4. VP4" [8] (Figure 1).

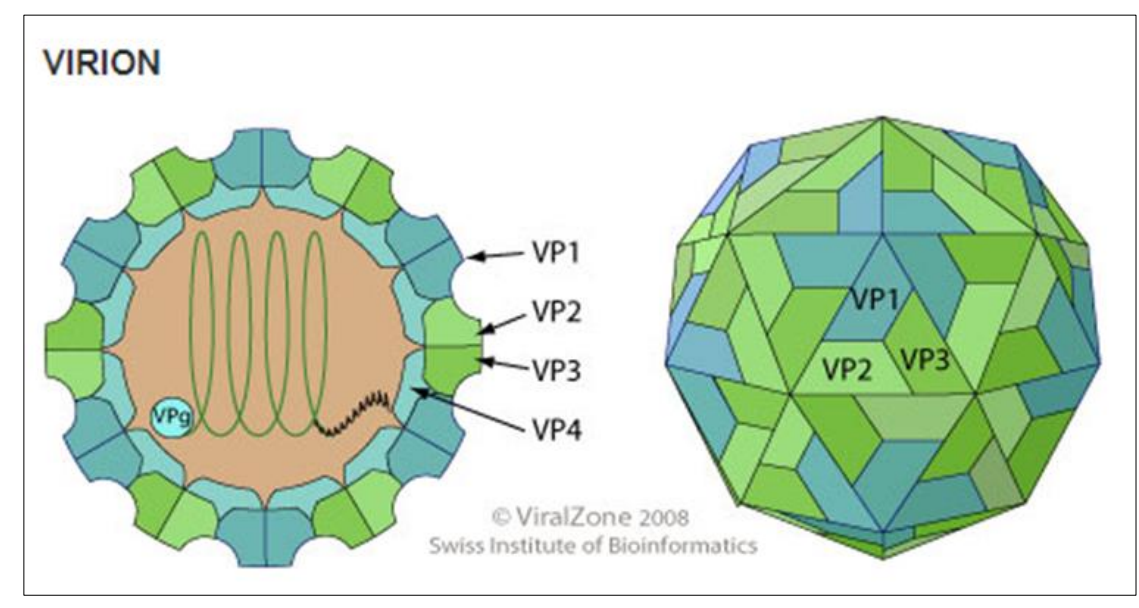

Figure 1 Viral structure of Aphthovirus [8]

\subsection{Viral genome of Aphthovirus and gene expression}

A single ORF encodes a polyprotein, polyadenylated linear ssRNA(+) genome of 7.5 to $8.5 \mathrm{~kb}$. At 5 ' end of the viral genomic RNA, a viral protein "VPg" is available. There is a type II internal ribosome entry site "IRES" at the 5 ' end of the long UTR. The structural polypeptides are encoded in the P1 region.

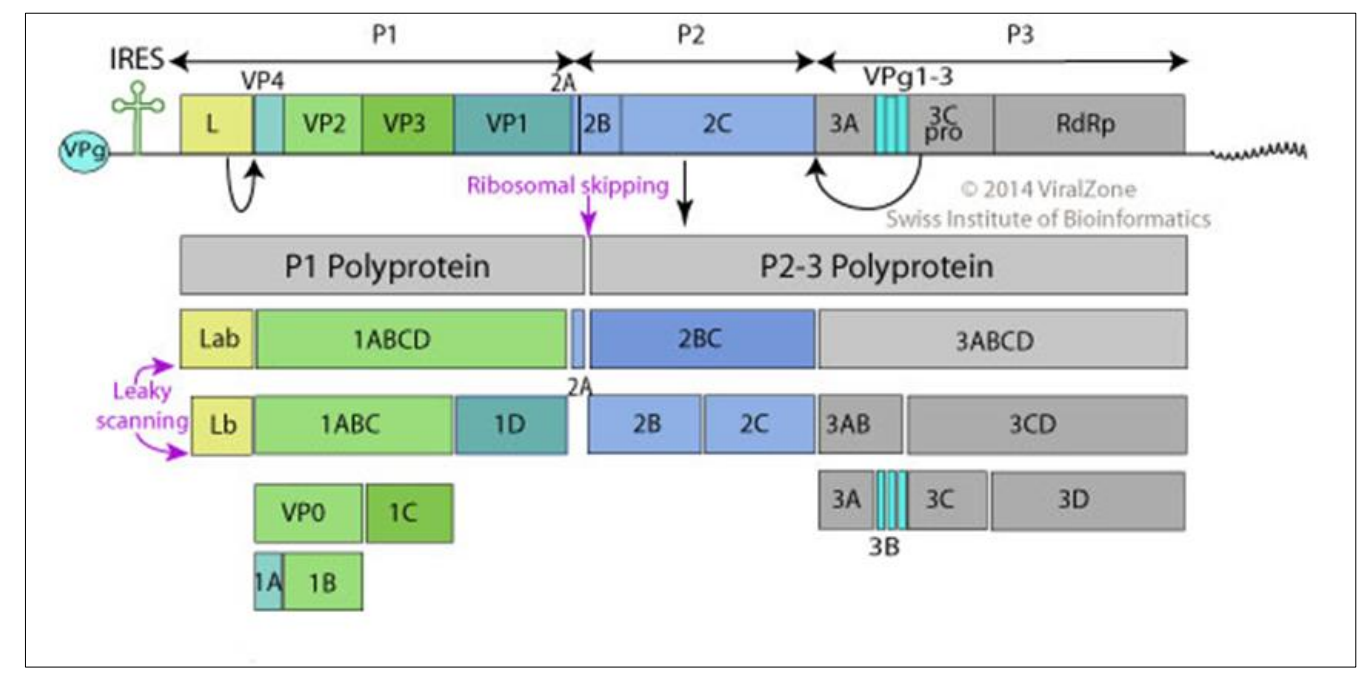

Figure 2 Viral genome of Aphthovirus [8] 
The replication of non-structural proteins is encoded by the domains of P2 and P3. In addition to the "3C protease" "L protease," this gene encodes a "N-terminal" leader protease. The shorter 3' UTR is required for (-) strand synthesis (Figure 2) [8].

The genome and viral messenger RNA are both provided by the RNA virion's gene expression. The IRES allows the polyprotein to be immediately translated. Viral proteases break down the polyprotein into many precursor and mature proteins, which produce structural and non-structural proteins like replicase. The host cell is modified by the viral structure and non-structure proteins, this results in cell lysis. At the point where the $2 \mathrm{~A}$ and downstream sequences intersect, ribosomal skipping is used [9].

\subsection{Viral replication}

Viruses reproduce in the cytoplasm. The steps for viral replication are as follows: The virus's attachment to host receptors causes viral endocytosis in the host cell via clathrin-dependent endocytosis. When the capsid is acidified, it produces VP4, which allows the viral genomic RNA in the hot cell cytoplasm to open a pore in the endosomal membrane of the host. The capsid is split into pentameric subunits when exposed to acidic PH. The polyprotein VPg is generated from viral RNA and subsequently translated. Viral protease cleavage closes the cellular cap-dependent translation of translation factors. Replication takes place in ER membrane vesicles, which are viral factories. The ssRNA(+) genome is used to make the dsRNA genome. New ssRNA(+) and viral mRNAs are produced when the dsRNA genome is replicated/transcribed. Preassembled procapsids are thought to contain new genomic RNA. The virus is released during cell lysis. Maturation of an unknown host protease (figure 3) [8,9].

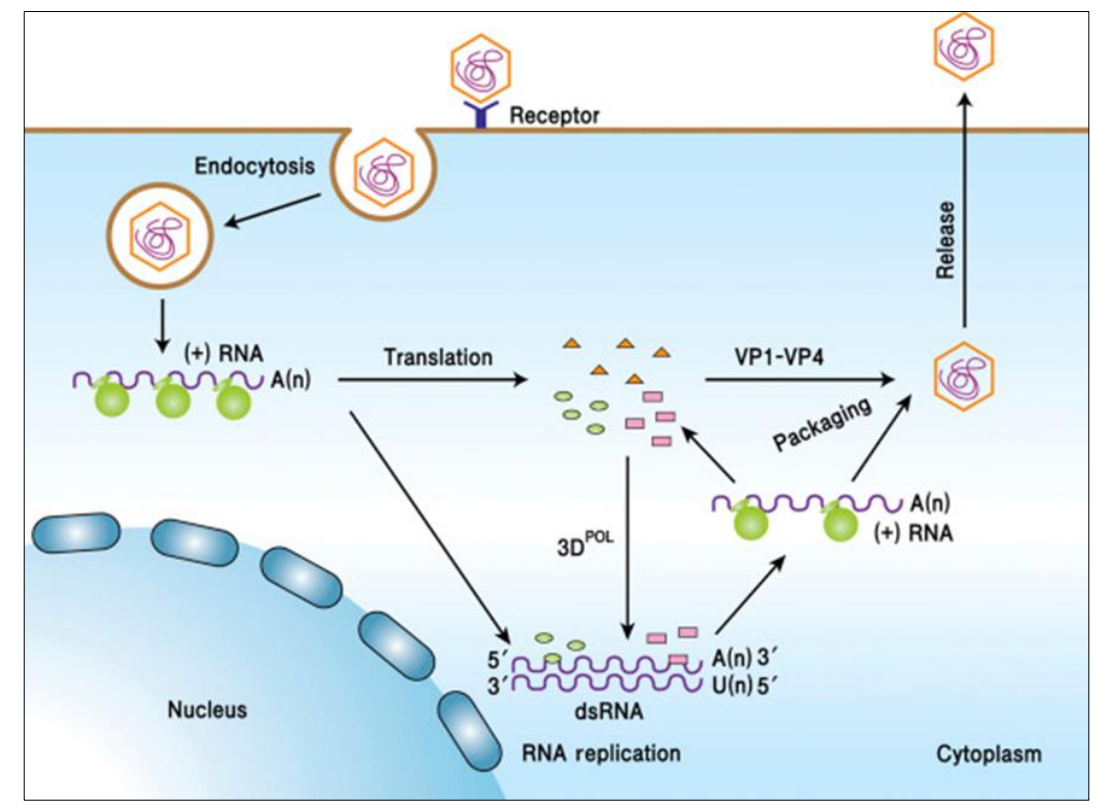

Figure 3 Viral replication of Aphthovirus [9]

\section{Epidemiology of foot mouth disease}

FMD has a complex epidemiology. A number of viral, host, or environmental factors influences it, including as changes in virulence, particle stability, and the chance of long-term persistence in different microenvironments. The multiplication and spread of FMDV are all impacted by host immunity, nutrition and species, animal movement, population density, links among different wild and domestic animals that are able to distribute the virus mechanically. While adequate meteorological conditions are prevalent, the environment can present geographical impediments to the transmission of the virus or offer biological barriers to viral communication $[10,11]$.

The main route of virus entrance into spontaneous infections is through the respiratory tract. In the pharyngeal epithel, the initial proliferation of the virus normally occurs and produces primary vesicles or "aphthaes." Depending on the host species and on the virus strain which they are infected, the clinical outcomes may differ. Sick animals, especially pigs, are commonly expanded by high viruses, and 1012 infectious units have been documented per infected animal. In ruminants prolonged asymptomatic infection may occur, and the animal's esophagus and throat fluids can be removed from an infectious virus from a matter of weeks to several years following initial infection [12]. 


\section{Pathogenesis of foot mouth disease}

Inhalation is the most excellent strategy of infecting the tissues although viruses also come into touch with abraded skin through eating, insemination and inoculation. After inhalation, the pharyngeal mucosa and lymphtic tissues develop primary viral replication [13]. Viraemia is a result of the primary diagnosis; lymph nodes, mammalian glands and other organs as well as epithelial cells of the mouth, mouth, mouth, teats, skin and coronary bands are the result of viral replication (figure 4) (13). Although peptides containing the RGD were able to inhibit parental virus interaction with integrated molecules, the existence of such integrative peptides had not inhibited the infectivity of FMDV isolates adapted to the cell cultivation, indicating a shift between an RGD-related RGD-dependent adhesion site mechanism. On the other hand, FMDV isolates acclimated to cells culture " their ability to engage with integrins is preserved"[14]. This is an important component of the numerous anticorruption epitopes known. The absence, however, of a motif for cell entrance that is integrated with RGD significantly increases FMDV's antigenic variations and encourages the isolation from radically changed antigenic mutants containing RGD triplet mutations [13-14].

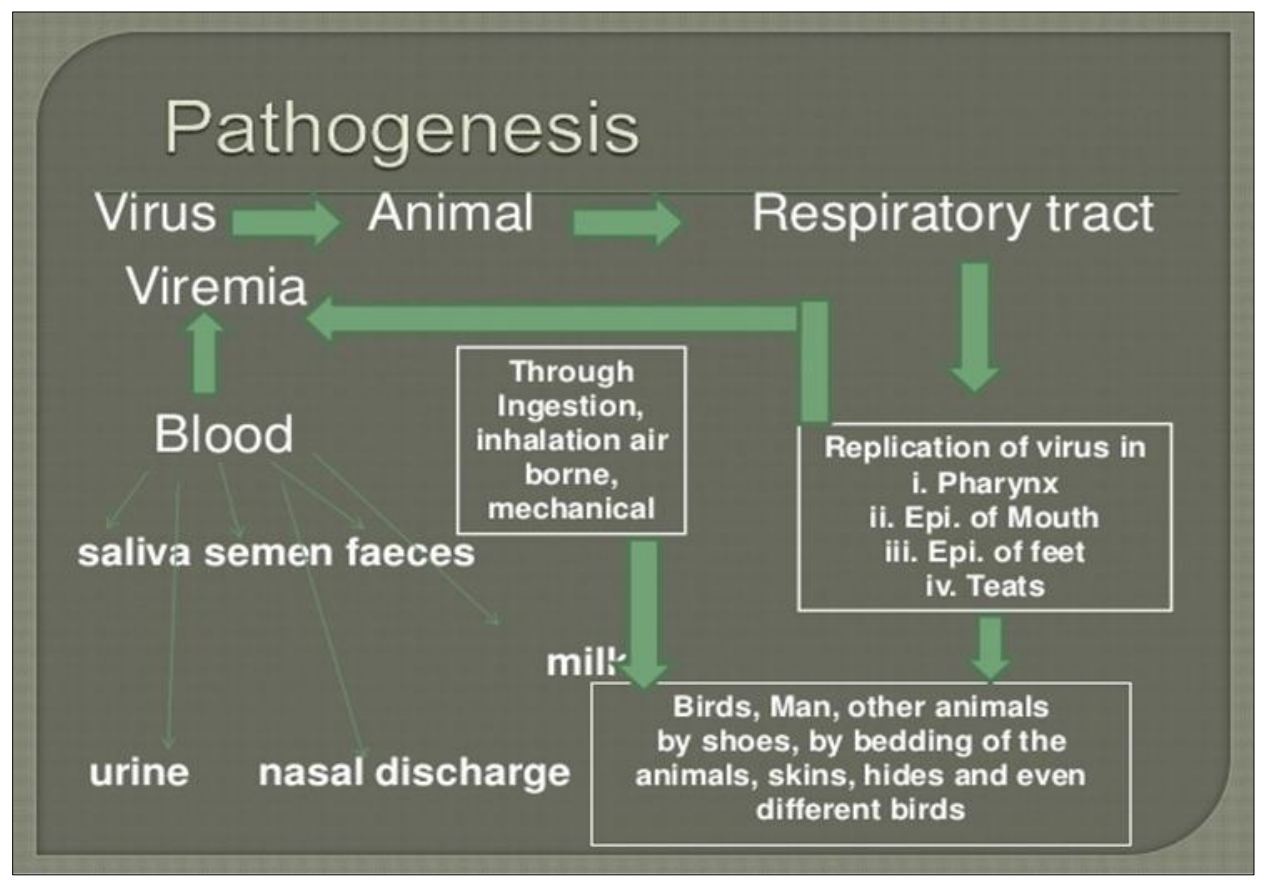

Figure 4 Pathogenesis of foot mouth disease [13]

The access of the integrated site to the neutralization of the antigens indicates a coevolution of FMDV antigenicity and the use of the receptor. The genetic modifications FMDV could have in the employment of alternative mechanisms for cell identification are minimal. On the other hand, in the mutant FMDV spectrum propagating in the animal host viruses with extraordinary receptor binding properties are most likely to exist [15]. The result of the selection of antigenic variant anti-FMDV antibiotic anticorrosion in peptide-vaccinated livestock suggested that these particular in vivo FMDV mutant diseases occur. The gradual prevalence of an unanticipated amino acid replacement that has affected the antigenic structure of the G-H loop of capsid protein VP1, throughout FMDV adaptations, has been shown in a recently study examined the genetic modifications selected throughout the FMDV adaptation to Guinea Pig [14]. FMDV cDNA experiments showed that this mutation was needed for viral contact with BHK receptor molecules as well as other regularly utilized cell lines for FMDV proliferation. As noted above, the identification of the mutant of FMDV that showing modification in their antigens and resulted change at cell tropism of different animals [13-15]. The severity of the "FMDV"-induced symptoms varies depending on the species afflicted, as well as the disease's dose or hereditary features. The concept of a host spectrum is founded on "FMDV's" ability to generate clinical symptoms, and failure to do so does not negate "FMDV's" ability to replicate in a specific species $[9,12]$.

"Non-structural protein of virus may be deletion at 3A leading to attenuation of "FMDV" serotype "C" and "O" in cattle following passages in chicken embryos. The deletion of overlapping (10 amino acids) or point mutations at $3 \mathrm{~A}$ have demonstrated that the poor pathogenicity of an "FMDV" serotype O isolated variant was observe in 1997 in Taiwan [16]. It was interesting to note that this virus was very virulent in swine, showing both that species was frequently connected to attenuating FMDV as well as the intricate interactions between the virus and the host that characterize the 
infection progression toward lesions and clinical signs. In addition, the research of the molecular base of "FMDV" adaption to the pig has shown information on the involvement of 3A protein of virus [17]. The FMDV and other picornaviruses are currently shown to result in complicated dynamics caused by diverse selective pressures by the interaction between quasi-specific FMDV and different hosts [12, 17].

\subsection{Clinical signs of foot mouth disease}

The FMDV incubation time is 1-12 days incubation time. There might be several clinical indications of foot-and-mouth disease in cattle (FMD). Symptoms of FMD in cattle include pyrexia and anorexia, and decreased milk output in dairy animals for 2-3 days [18]. Vesicles form also on the mouth and nasal mucous membrane and on the coronary band between the claws [19]. The result is lip snapping, bruxism, blowjob, lameness, stamping or kicking of feet. In addition, on the mammary glands, vesicles often develop. Erosions appear 24 hours later after the vesicle rupture [20]. In 8-15 days on average the cattle recover from FMD, however there may be difficulties. The problems include secondary infections of injuries, decreased milk supply, loss of weight, tongue erosions, hoof distortions, abortion and mastitis. Young animals may nevertheless succeed in myocarditis (figure 5) [18-20].

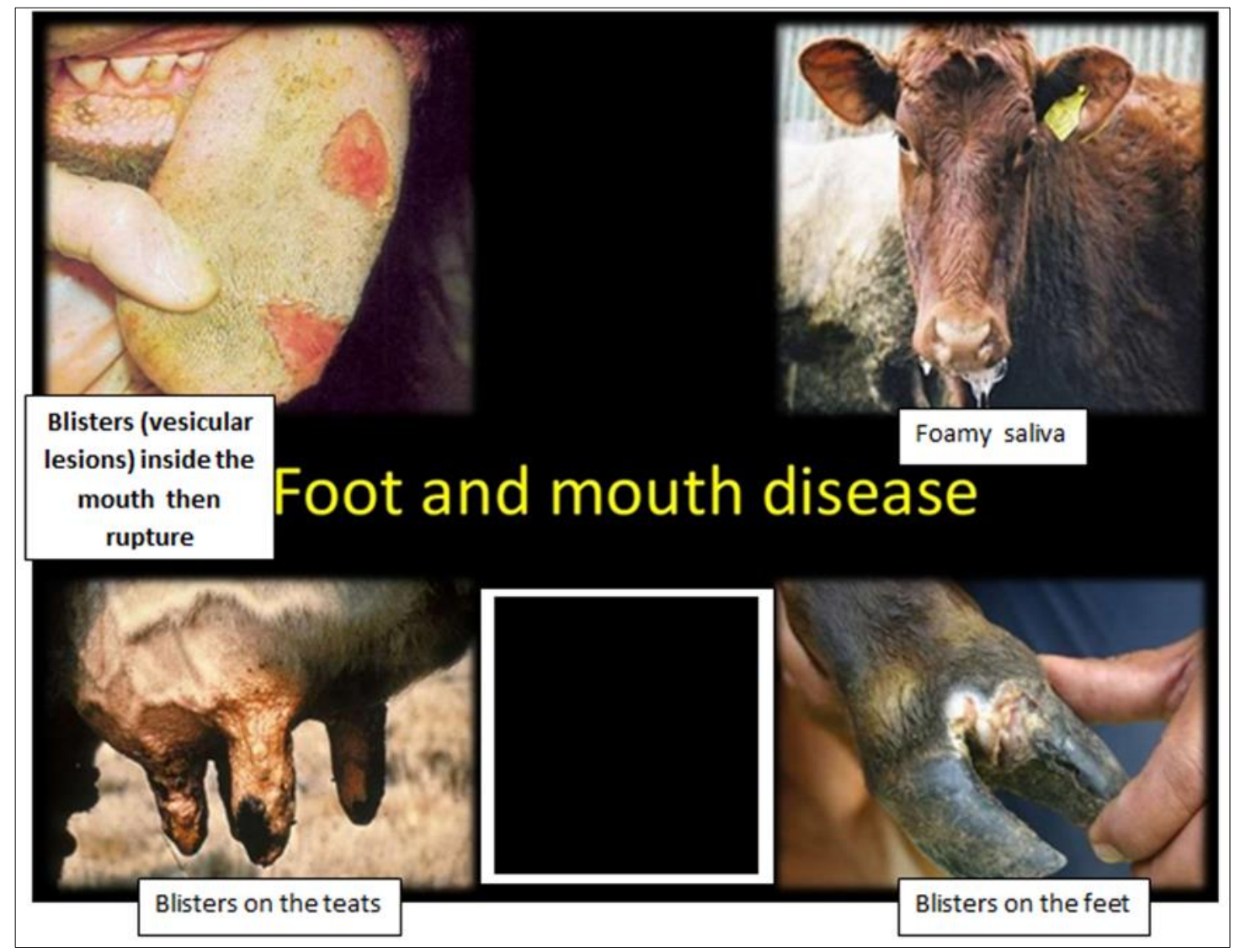

Figure 5 Sings of foot mouth disease [20]

\subsection{Immune response of foot mouth disease}

FMDV causes quick humoral reactions in infected animals and provides good protection against reinfection with antigenically-related and homologous viruses. FMD protection is related to the development of high quantities of neutralizing serum antibodies. Following viral opsonisation, the proposal was made to mediate the viral clearance of virus-antibody complexes [21]. Neutralizing antibodies can be identified quickly after FMDV infection or immunization with viral capsid B cell epitopes. Within 2 weeks after immunization after IgM synthesis, IgGs become the most important neutralizing antibodies within 3-4 days of infection or vaccination [22]. The response of IgG1 is generally more than the response of IgG2. In the upper respiratory and gastrointestinal secretions soon after infection or immunization, there is a detectable antibody response [23]. The influence on the "defense of innate immune" response in immunized animals with "FMDV" is becoming significant together with the attitudes to success in vaccine efforts. FMDV and immunization both produce distinct T-cell responses (Figure 6) [24]. 
The predominantly T-cell-mediated lymphoproliferative response "CD4+" involves activation of the B cell, and the synthesis of antimicrobials in animals and pigs [23]. These T-helping cells that define a wide range of non-structural proteins and viral epitopes in capsid are probable to be important to support FMDV by encouraging antibody production and producing an environment that is perfect for synergistic immune response. FMDV infection produces a fast decrease on the surface of vulnerable cells, in the expression of MHC class I. This outcome could impede the ability of the infected cells to provide cytotoxic T-cells (CTLs) with viral peptides allowing the host to evade the CTL response [22, 24].

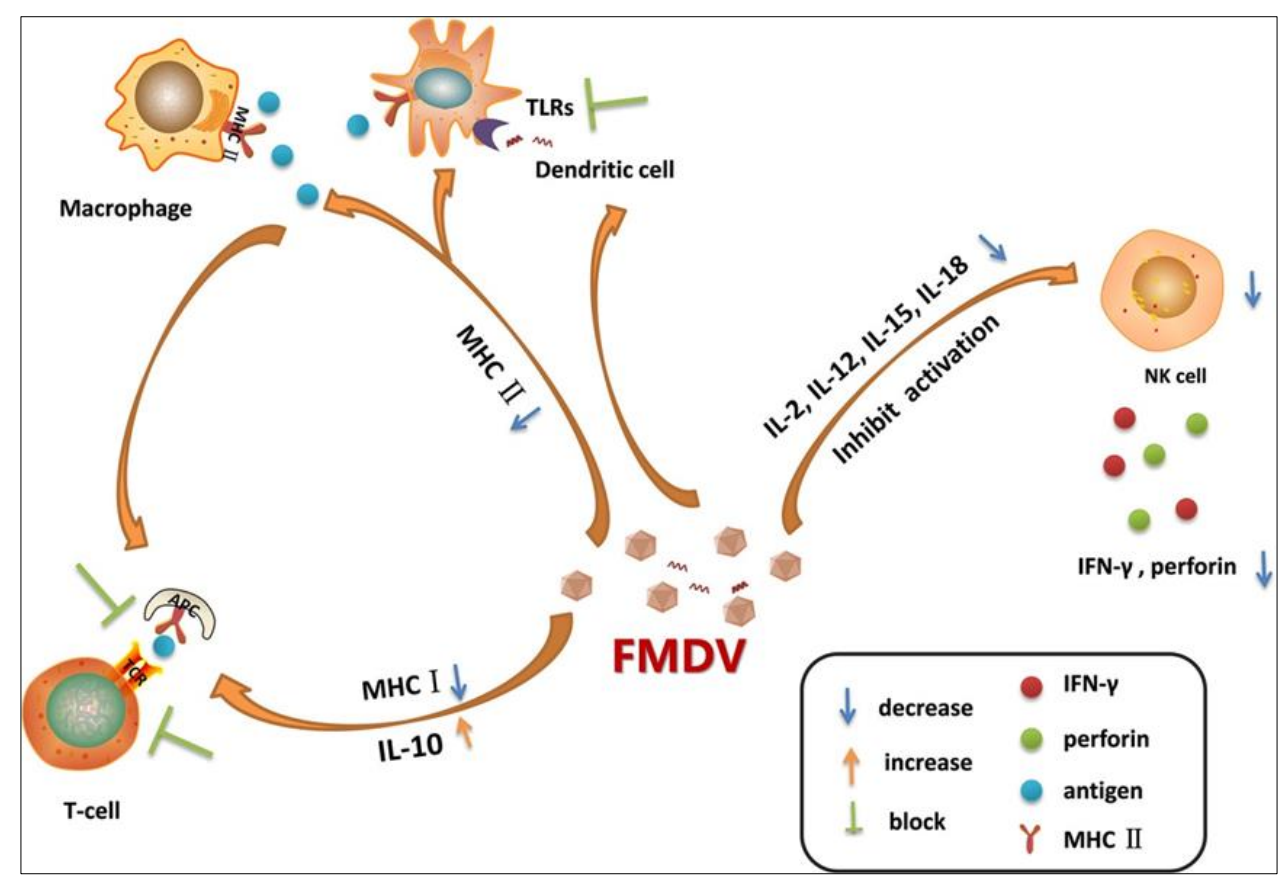

Figure 6 Immune response to foot mouth disease (24)

\section{Diagnosis of foot mouth disease}

\subsection{Direct diagnosis (Antigen detection)}

\subsubsection{Viral isolation}

Frenkel initially described the "FMDV" isolation of the main epithel cells of the bovine tongue. The main principal bovine and pig's kidney cells are adapted to diagnose FMDV by Salesmen and Bachrach et al. Bovine thyroid cells were historically considered to be "FMDV's" best primary cells. However, continuous cell lines were created more recently. Bovine kidney cells expressed $\beta 6$ and $V$ and integrated subunits, among multiple stable cells, were particularly sensitive to all FMDV serotypes [25-27].

\subsubsection{Complement fixation test}

This approach depends on the fact that the complexes of viral antibodies bind the complement of Guinea pigs. In the absence of virus antibody binding in the presence of RBC anti-sheep, the free supplement lyses sheep-red blood cells [25]. Due to the serotype-specific nature of FMDV antibodies, direct supplement fixation testing was performed to identificate FMDV serotypes [26].

\subsubsection{ELISA test}

Antigen capture enzyme immune sorbent assay (ELISA) are currently available based on monoclonal or polyclonal antibodies used to detection the structural or non- structural proteins. ELISA can examine a large number of samples [27]. 


\subsubsection{Lateral Flow test}

Is an antigen-capture assays or chromatographic band tests permit swift diagnosis on the spot when a quick result is necessary in endemic areas and reference laboratories. These tests detect antigens of FMDV in vesicular fluids or epithelial suspensions from infected animals using monoclonal or polyclonal antibodies [27].

\subsubsection{Reverse transcription-polymerase chain reaction (RT-PCR)}

Is the test used to identified the nucleic acid of infectious viruses in addition to diagnosis the inactivated viruses, which reduce the impact of virus sample treatment defects? RT-PCR real-time was commonly utilized to diagnose FMDV due to enhanced analytical sensitivity and ease of usage [28].

\subsection{Indirect diagnosis (Antibody detection)}

\subsubsection{Complement fixation test (Indirect)}

The World Organization for Animal Health (OIE) recommends that only if the FMDV ELISA test is not available is the indirect additional fixation test [25, 29].

\subsubsection{Neutralization test}

Is a serum-virus interaction test and is a particular serotype test used to detect neutralizing vaccine or infection-induced antibodies. The presence of neutralizing circulating antibodies is predominantly linked to viremia resolution [29].

\subsubsection{ELISA test (Indirect)}

This test used to identify the antibody for virus serotyping and several FMDV. Two kinds of ELISA assays are available: ELISA structural protein and ELISA non-structural protein. Viral structural protein ELISA is designed to detect vaccination or infection-induced antibodies against capsid protein with polyclonal or monoclonal antigens (VP1, VP2, and VP3). However, ELISA has been employed in FMDV target antigens in viral non-structural proteins such as (2A, 2B $2 \mathrm{C})$ and $(3 \mathrm{ABC}, 3 \mathrm{AB}, 3 \mathrm{~B}, 3 \mathrm{C})$. Samples of serum FMDV exposed from unvaccinated and vaccinated cattle, piglets and sheep were examined for the OIE index screening [25, 26, 29].

\subsection{Vaccines}

\subsubsection{Conventional vaccines}

These vaccinations are relied on inactivated chemical viruses. Viruses of different serotypes are often added into the formulations of vaccines. Quality-tested vaccinations, customized to the circulating viruses, including antigenic composites are necessary for efficient "FMD" protection. Inactivated vaccinations generate a consistent, but usually weaker, humoral response than that elicited by infected animals [30].

\subsubsection{Viral protein and peptide vaccines}

Early findings revealed VP1 or half segments of viral capsid components able to neutralize antibodies with incomplete protection. Recombinant VP1 has immunogenicity as viral particles [30]. The capside preserves is most antigenic and immunogenic characteristics of virus particles. P1 polypeptide expression, however, created a modest quantity of empty virus capsids in vaccines and baculoviruses that require the necessary 3C P1 processing. The expression of virus protein in vector and DNA replication vaccines The development of recombinant replicating Vector Viral Immunogens is an approach for generating the vast range of immune responses that occur during viral infection in an effective way [31].

\subsubsection{Genetically engineered attenuated strains}

The attenuated viruses of virulent forms $[32,33]$.

\section{Conclusion}

From this review, we concluded the foot and mouth disease remained a highly contagious, infectious disease and distributed in a wide geographical area. As well, the virus was diagnosed via several tests. Along with this, the food and agriculture organization had proposed for controlling the disease via several types of vaccines. In future, research must be focused on the explain in detail the diagnostic tools and mechanism of vaccines to prevent the disease. 


\section{Compliance with ethical standards}

\section{Acknowledgments}

The authors owe sincere thanks to Aida B. Allawe for her support to us during the writing of this review.

\section{Disclosure of conflict of interest}

All authors declare that they have no conflict of interest.

\section{References}

[1] Kim ES, Saw J, Kadian-Dodov D, Wood M, Ganesh SK. FMD and SCAD: sex-biased arterial diseases with clinical and genetic pleiotropy. Circulation Research. 2021; 128(12): 1958-1972.

[2] Yi C, Yang Q, Scoglio CM. Understanding the effects of contact structures and information sharing on the FMD transmission among beef cattle farms. bioRxiv. 2021; 2020-04.

[3] Krishnaswamy N, Jeyakumar S, Selvan RT, Gowane GR, Mahadappa P, Vijayapillai U, Sanyal A. Short-term effect of foot-and-mouth disease (FMD) vaccination on the milk yield in the Deoni and crossbred cows. Tropical Animal Health and Production. 2021; 53(2): 1-6.

[4] Park SH, Lee SY, Kim JS, Kim AY, Park SY, Lee JH, Ko YJ. Scale-Up Production of Type 0 and A Foot-and-Mouth Disease Bivalent Vaccine and Its Protective Efficacy in Pigs. Vaccines. 2021; 9(6): 586.

[5] Lazarus DD, Opperman PA, Sirdar MM, Wolf TE, van Wyk I, Rikhotso OB, Fosgate GT. Improving foot-and-mouth disease control through the evaluation of goat movement patterns within the FMD protection zone of South Africa. Small Ruminant Research. 2021; 106448.

[6] Khan S, Shah SAA, Jamal SM. Evaluation of Sandwich Enzyme-Linked Immunosorbent Assay and Reverse Transcription Polymerase Chain Reaction for the Diagnosis of Foot-and-Mouth Disease. Intervirology. 2021; 1-6.

[7] Satsook P, Rattanatabtimtong S, Piasai L, Towiboon P, Somgird C, Pinyopummin A. Ovarian activity in crossbred Thai native does during naturally occurring foot-and-mouth disease (FMD) virus infection. Tropical Animal Health and Production. 2021; 53(2): 1-6.

[8] Jackson T, Belsham GJ. Picornaviruses: A View from 3A. Viruses. 2021; 13: 456.

[9] Yi J, Peng J, Yang W, Zhu G, Ren J, Li D, Zheng H. Picornavirus 3C-a protease ensuring virus replication and subverting host responses. Journal of Cell Science. 2021; 134(5): jcs253237.

[10] Dabasa G, Abunna F. Review on Epidemiology of Foot and Mouth Disease (FMD) in Ethiopia. J Trop Dis. 2021; 9 : 269.

[11] László Z, Pankovics P, Reuter G, Cságola A, Bálint Á, Albert M, Boros Á. Multiple Types of Novel Enteric Bopiviruses (Picornaviridae) with the Possibility of Interspecies Transmission Identified from Cloven-Hoofed Domestic Livestock (Ovine, Caprine and Bovine) in Hungary. Viruses. 2021; 13(1): 66.

[12] Suchowski M, Eschbaumer M, Teifke JP, Ulrich R. After nasopharyngeal infection, foot-and-mouth disease virus serotype A RNA is shed in bovine milk without associated mastitis. Journal of Veterinary Diagnostic Investigation. 2021.

[13] Zahid MN, Shahid MA, Imran HM, Oneeb M, Ahmed M, Rehman ZU, Yaqub T. Integrins and Heparan Sulfate Play Crucial Role in Pathogenesis of Foot-and-Mouth Disease Virus. Foot. 2020.

[14] Zhang Z, Waters R, Li Y. Pathogenesis of non-epithelial foot-and-mouth disease in neonatal animals. Veterinary Microbiology. 2020; 108961.

[15] Xu Y, Zheng Y, Shi W, Guan L, Yu P, Xu J, Xu J. Pathogenic characteristics of hand, foot and mouth disease in Shaanxi Province, China, 2010-2016. Scientific reports. 2020; 10(1): 1-11.

[16] Ramulongo TD, Maree FF, Scott K, Opperman P, Mutowembwa P, Theron J. Pathogenesis, biophysical stability and phenotypic variance of SAT2 foot-and-mouth disease virus. Veterinary microbiology. 2020; 243: 108614.

[17] Cañas Arranz R. Synthetic dendrimer peptide vaccines against Foot-and-Mouth Disease Virus. 2020. 
[18] Awel SM, Dilba GM, Abraha B, Zewde D, Wakjira BS, Aliy A. Seroprevalence and Molecular Detection of Foot and Mouth Disease Virus in Dairy Cattle Around Addis Ababa, Central Ethiopia. Veterinary Medicine: Research and Reports. 2021; 12: 187.

[19] Arjkumpa O, Yano T, Prakotcheo R, Sansamur C, Punyapornwithaya V. Epidemiology and National Surveillance System for Foot and Mouth Disease in Cattle in Thailand during 2008-2019. Veterinary sciences. 2020; 7(3): 99.

[20] Rout M, Mohapatra JK. Foot-and-Mouth Disease: A Serious Threat for Dairy Industry. Biotica Research Today. 2021; 3(5): 360-363.

[21] de León P, Cañas-Arranz R, Saez Y, Forner M, Defaus S, Cuadra D, Hammer SE. Association of Porcine Swine Leukocyte Antigen (SLA) Haplotypes with B-and T-Cell Immune Response to Foot-and-Mouth Disease Virus (FMDV) Peptides. Vaccines. 2020; 8(3): 513.

[22] Li S, Yang Y, Lin X, Li Z, Ma G, Su Z, Zhang S. Biocompatible cationic solid lipid nanoparticles as adjuvants effectively improve humoral and $\mathrm{T}$ cell immune response of foot and mouth disease vaccines. Vaccine. 2020; 38(11): 2478-2486.

[23] Lu Y, Dong H, Li J, Li L, Wang M, Liu H, Sun S. Enhanced protective immune response of foot-and-mouth disease vaccine through DNA-loaded virus-like particles. Microbial pathogenesis. 2020; 143: 104130.

[24] Kim H, Kim AY, Choi J, Park SY, Park SH, Kim JS, Ko, YJ. Foot-and-Mouth Disease Virus Evades Innate Immune Response by 3C-Targeting of MDA5. Cells. 2021; 10(2): 271.

[25] Wong CL, Yong CY, Ong HK, Ho KL, Tan WS. Advances in the Diagnosis of Foot-and-Mouth Disease. Frontiers in veterinary science. 2020; 7: 477.

[26] Hwang YJ, Lee KK, Kim JW, Chung KH, Kim SJ, Yun WS, Lee CS. Effective Diagnosis of Foot-And-Mouth Disease Virus (FMDV) Serotypes 0 and A Based on Optical and Electrochemical Dual-Modal Detection. Biomolecules. 2021; 11(6): 841.

[27] Karniely S, Hamed F, Gelman B, King R, Storm N, Eyngor E, Even Tov B. First isolation of foot and mouth disease virus from wild boars in the Middle East. Transboundary and emerging diseases. 2020; 67(4): 1725-1729.

[28] Wang Y, Das A, Zheng W, Porter E, Xu L, Noll L, Bai J. Development and evaluation of multiplex real-time RT-PCR assays for the detection and differentiation of foot-and-mouth disease virus and Seneca Valley virus 1. Transboundary and emerging diseases. 2020; 67(2): 604-616.

[29] Liu W, Shao J, Zhang G, Chang Y, Ge S, Sun Y, Chang H. Development of an Indirect Chemiluminescence Immunoassay Using a Multiepitope Recombinant Protein To Specifically Detect Antibodies against Foot-andMouth Disease Virus Serotype $O$ in Swine. Journal of Clinical Microbiology. 2020; 59(3): e02464-20.

[30] Hardham JM, Krug P, Pacheco JM, Thompson J, Dominowski P, Moulin V, Rieder E. Novel foot-and-mouth disease vaccine platform: formulations for safe and DIVA-compatible FMD vaccines with improved potency. Frontiers in Veterinary Science. 2020; 7.

[31] Avendaño C, Celis-Giraldo C, Ordoñez D, Díaz-Arévalo D, Rodríguez-Habibe I, Oviedo J, Patarroyo MA. Evaluating the immunogenicity of chemically-synthesised peptides derived from foot-and-mouth disease VP1, VP2 and VP3 proteins as vaccine candidates. Vaccine. 2020; 38(23): 3942-3951.

[32] Hao R, Ma K, Ru Y, Li D, Song G, Lu B, Zheng H. Amber codon is genetically unstable in generation of premature termination codon (PTC)-harbouring Foot-and-mouth disease virus (FMDV) via genetic code expansion. RNA biology. 2021; 1-12.

[33] Eschbaumer M, Dill V, Carlson JC, Arzt J, Stenfeldt C, Krug PW, Rieder E. Foot-and-mouth disease virus lacking the leader protein and containing two negative DIVA markers (FMDV LL3B3D A24) is highly attenuated in pigs. Pathogens. 2020; 9(2): 129. 